\title{
Comparative growth measures of differential monomials and differential polynomials depending on their relative orders
}

\author{
Sanjib Kumar Datta ${ }^{1}$, Tanmay Biswas ${ }^{2}$, Debasmita Dutta ${ }^{3}$ \\ ${ }^{1}$ Department of Mathematics, University of Kalyani, P.O.-Kalyani, Dist.-Nadia, PIN, West Bengal, India \\ ${ }^{2}$ Rajbari, Rabindrapalli, R. N. Tagore Road, P.O.-Krishnagar, Dist.-Nadia, PIN-741101, West Bengal, India \\ ${ }^{3}$ Mohanpara Nibedita Balika Vidyalaya (HIgh), P.O.-Amrity, Block-English Bazar, Dist.-Malda, PIN, West Bengal, India.
}

Received: 6 June 2016, Accepted: 14 August 2016

Published online: 26 December 2016.

\begin{abstract}
In the paper we establish some new results depending on the comparative growth properties of composite entire or meromorphic functions using relative order ( relative lower order ) and differential monomials, differential polynomials generated by one of the factors.
\end{abstract}

Keywords: Entire function, meromorphic function, order (lower order ), relative order (relative lower order ), Property (A), growth, differential monomial, differential polynomial.

\section{Introduction, definitions and notations}

We denote by $\mathbb{C}$ the set of all finite complex numbers. Let $f$ be a meromorphic function defined on $\mathbb{C}$. We use the standard notations and definitions in the theory of entire and meromorphic functions which are available in [22] and [?].

Let $f$ be a non-constant meromorphic function defined in the open complex plane $\mathbb{C}$. Also let $n_{0 j}, n_{1 j}, \ldots n_{k j}(k \geq 1)$ be non-negative integers such that for each $j, \sum_{i=0}^{k} n_{i j} \geq 1$. We call $M_{j}[f]=A_{j}(f)^{n_{0 j}}\left(f^{(1)}\right)^{n_{1 j}} \ldots\left(f^{(k)}\right)^{n_{k j}}$ where $T\left(r, A_{j}\right)=S(r, f)$ to be a differential monomial generated by $f$. The numbers $\gamma_{M j}=\sum_{i=0}^{k} n_{i j}$ and $\Gamma_{M j}=\sum_{i=0}^{k}(i+1) n_{i j}$ are called respectively the degree and weight of $M_{j}[f]\{[5],[26]\}$. The expression $P[f]=\sum_{j=1}^{s} M_{j}[f]$ is called a differential polynomial generated by $f$. The numbers $\gamma_{P}=\max _{1 \leq j \leq s} \gamma_{M j}$ and $\Gamma_{P}=\max _{1 \leq j \leq s} \Gamma_{M j}$ are called respectively the degree and weight of $P[f]\{[5],[26]\}$. Also we call the numbers $\gamma_{P}=\min _{1 \leq j \leq s} \gamma_{M j}$ and $k$ (the order of the highest derivative of $f$ ) the lower degree and the order of $P[f]$ respectively. If $\gamma_{p}=\gamma_{P}, P[f]$ is called a homogeneous differential polynomial. Throughout the paper we consider only the non-constant differential polynomials and we denote by $P_{0}[f]$ a differential polynomial not containing $f$ i.e., for which $n_{0 j}=0$ for $j=1,2, \ldots s$. We consider only those $P[f], P_{0}[f]$ singularities of whose individual terms do not cancel each other. We also denote by $M[f]$ a differential monomial generated by a transcendental meromorphic function $f$.

In the sequel the following definitions are well known. 
Definition 1. Let a be a complex number, finite or infinite. The Nevanlinna deficiency and the Valiron deficiency of ' $a$ ' with respect to a meromorphic function $f$ are defined as

$$
\delta(a ; f)=1-\limsup _{r \rightarrow \infty} \frac{N_{f}(r, a)}{T_{f}(r)}=\liminf _{r \rightarrow \infty} \frac{m_{f}(r, a)}{T_{f}(r)}
$$

and

$$
\Delta(a ; f)=1-\liminf _{r \rightarrow \infty} \frac{N_{f}(r, a)}{T_{f}(r)}=\limsup _{r \rightarrow \infty} \frac{m_{f}(r, a)}{T_{f}(r)} .
$$

Definition 2. The quantity $\Theta(a ; f)$ of a meromorphic function $f$ is defined as follows

$$
\Theta(a ; f)=1-\limsup _{r \rightarrow \infty} \frac{\bar{N}_{f}(r, a)}{T_{f}(r)}
$$

Definition 3. [29] For $a \in \mathbb{C} \cup\{\infty\}$, we denote by $n_{f \mid=1}(r, a)$, the number of simple zeros of $f-a$ in $|z| \leq r . N_{f \mid=1}(r, a)$ is defined in terms of $n_{f \mid=1}(r, a)$ in the usual way. We put

$$
\delta_{1}(a ; f)=1-\limsup _{r \rightarrow \infty} \frac{N_{f \mid=1}(r, a)}{T_{f}(r)}
$$

the deficiency of ' $a$ ' corresponding to the simple a-points of $f$ i.e., simple zeros of $f-a$.

Yang [28] proved that there exists at most a denumerable number of complex numbers $a \in \mathbb{C} \cup\{\infty\}$ for which $\delta_{1}(a ; f)>0$ and $\sum_{a \in \mathbb{C} \cup\{\infty\}} \delta_{1}(a ; f) \leq 4$.

Definition 4. [24] For a $\varepsilon \mathbb{C} \cup\{\infty\}$, let $n_{p}(r, a ; f)$ denote the number of zeros of $f-a$ in $|z| \leq r$, where a zero of multiplicity $<p$ is counted according to its multiplicity and a zero of multiplicity $\geq p$ is counted exactly $p$ times and $N_{p}(r, a ; f)$ is defined in terms of $n_{p}(r, a ; f)$ in the usual way. We define

$$
\delta_{p}(a ; f)=1-\limsup _{r \rightarrow \infty} \frac{N_{p}(r, a ; f)}{T_{f}(r)} .
$$

Definition 5. [3] $P[f]$ is said to be admissible if

(i) $P[f]$ is homogeneous, or

(ii) $P[f]$ is non homogeneous and $m_{f}(r)=S_{f}(r)$.

During the past decades, several authors ( see [6] to [17], [25]) made closed investigations on comparative study of the growth properties of composite entire or meromorphic functions in different directions using order (lower order ) and differential polynomials and differential monomials generated by one of the factors. The growth indicator such as order (lower order ) of entire or meromorphic function which is generally used in computational purpose is defined in terms of the growth of that function with respect to the exponential function is shown in the following definition:

Definition 6. The order $\rho_{f}$ ( the lower order $\lambda_{f}$ ) of an entire function $f$ is defined as

$$
\begin{gathered}
\rho_{f}=\limsup _{r \rightarrow \infty} \frac{\log \log M_{f}(r)}{\log \log M_{\exp z}(r)}=\limsup _{r \rightarrow \infty} \frac{\log \log M_{f}(r)}{\log (r)} \\
\left(\lambda_{f}=\liminf _{r \rightarrow \infty} \frac{\log \log M_{f}(r)}{\log \log M_{\exp z}(r)}=\liminf _{r \rightarrow \infty} \frac{\log \log M_{f}(r)}{\log (r)}\right) .
\end{gathered}
$$


when $f$ is a meromorphic, one may easily prove that

$$
\begin{gathered}
\rho_{f}=\limsup _{r \rightarrow \infty} \frac{\log T_{f}(r)}{\log T_{\exp z}(r)}=\limsup _{r \rightarrow \infty} \frac{\log T_{f}(r)}{\log \left(\frac{r}{\pi}\right)}=\limsup _{r \rightarrow \infty} \frac{\log T_{f}(r)}{\log (r)+O(1)} \\
\left.\lambda_{f}=\liminf _{r \rightarrow \infty} \frac{\log T_{f}(r)}{\log T_{\exp z}(r)}=\liminf _{r \rightarrow \infty} \frac{\log T_{f}(r)}{\log \left(\frac{r}{\pi}\right)}=\liminf _{r \rightarrow \infty} \frac{\log T_{f}(r)}{\log (r)+O(1)}\right) .
\end{gathered}
$$

Both entire and meromorphic function have regular growth if their order coincides with their lower order.

For a non-constant entire function $f, M_{f}(r)$ and $T_{f}(r)$ are both strictly increasing and continuous functions of $r$ and their inverses $M_{f}^{-1}(r):(|f(0)|, \infty) \rightarrow(0, \infty)$ and $T_{f}^{-1}:\left(T_{f}(0), \infty\right) \rightarrow(0, \infty)$ respectively exist where $\lim _{s \rightarrow \infty} M_{g}^{-1}(s)=\infty$ and $\lim _{s \rightarrow \infty} T_{f}^{-1}(s)=\infty$. In this connection we just recall the following definition which is relevant to our study:

Definition 7. [2] A non-constant entire function $f$ is said have the property (A) if for any $\sigma>1$ and for all sufficiently large $r,\left[M_{f}(r)\right]^{2} \leq M_{f}\left(r^{\sigma}\right)$ holds. For examples of functions with or without the Property $(A)$, one may see [2].

Bernal $\{[1],[2]\}$ initiated the idea of relative order of an entire function $f$ with respect to another entire function $g$, symbolized by $\rho_{g}(f)$ to keep away from comparing growth just with exp $z$ which is as follows:

$$
\begin{aligned}
\rho_{g}(f) & =\inf \left\{\mu>0: M_{f}(r)<M_{g}\left(r^{\mu}\right) \text { for all } r>r_{0}(\mu)>0 .\right\} \\
& =\limsup _{r \rightarrow \infty} \frac{\log M_{g}^{-1} M_{f}(r)}{\log r} .
\end{aligned}
$$

The definition agrees with the classical one [?] if $g(z)=\exp z$.

Similarly, one may define the relative lower order of an entire function $f$ with respect to another entire function $g$ symbolized by $\lambda_{g}(f)$ in the following way:

$$
\lambda_{g}(f)=\liminf _{r \rightarrow \infty} \frac{\log M_{g}^{-1} M_{f}(r)}{\log r} .
$$

Extending this idea, Lahiri and Banerjee [23] established the definition of relative order of a meromorphic function with respect to an entire function which is as follows:

Definition 8. [23] Let $f$ be any meromorphic function and $g$ be any entire function. The relative order of $f$ with respect to $g$ is defined as

$$
\begin{aligned}
\rho_{g}(f) & =\inf \left\{\mu>0: T_{f}(r)<T_{g}\left(r^{\mu}\right) \text { for all sufficiently large } r\right\} \\
& =\limsup _{r \rightarrow \infty} \frac{\log T_{g}^{-1} T_{f}(r)}{\log r} .
\end{aligned}
$$

Likewise, one may define the relative lower order of a meromorphic function $f$ with respect to an entire function $g$ in the following way:

$$
\lambda_{g}(f)=\liminf _{r \rightarrow \infty} \frac{\log T_{g}^{-1} T_{f}(r)}{\log r} .
$$

It is known \{cf. [23] $\}$ that if $g(z)=\exp z$ then Definition 8 coincides with the classical definition of the order of a meromorphic function $f$.

In the paper we prove some comparative growth properties of composite entire or meromorphic functions in almost a 
new direction in the light of their relative orders and relative lower orders and differential monomials, differential polynomials generated by one of the factor.

\section{Lemmas}

In this section we present some lemmas which will be needed in the sequel.

Lemma 1. [4] Let $f$ be meromorphic and $g$ be entire then for all sufficiently large values of $r$,

$$
T_{f \circ g}(r) \leqslant\{1+o(1)\} \frac{T_{g}(r)}{\log M_{g}(r)} T_{f}\left(M_{g}(r)\right) .
$$

Lemma 2. [18] Let $f$ be a meromorphic function and $g$ be an entire function such that $\lambda_{g}<\mu<\infty$ and $0<\lambda_{f} \leq \rho_{f}<\infty$. Then for a sequence of values of $r$ tending to infinity,

$$
T_{f \circ g}(r)<T_{f}\left(\exp \left(r^{\mu}\right)\right) .
$$

Lemma 3. [18] Let $f$ be a meromorphic function of finite order and $g$ be an entire function such that $0<\lambda_{g}<\mu<\infty$. Then for a sequence of values of $r$ tending to infinity,

$$
T_{f \circ g}(r)<T_{g}\left(\exp \left(r^{\mu}\right)\right) .
$$

Lemma 4. [21] Let $f$ be an entire function which satisfy the Property (A), $\beta>0, \delta>1$ and $\alpha>2$. Then

$$
\beta T_{f}(r)<T_{f}\left(\alpha r^{\delta}\right)
$$

Lemma 5. [19] If $f$ be a meromorphic function either of finite order or of non-zero lower order such that $\Theta(\infty ; f)=$ $\sum_{a \neq \infty} \delta_{p}(a ; f)=1$ or $\delta(\infty ; f)=\sum_{a \neq \infty} \delta(a ; f)=1$ and $g$ be an entire function of regular growth having non zero finite order and $\Theta(\infty ; g)=\sum_{a \neq \infty} \delta_{p}(a ; g)=1$ or $\delta(\infty ; g)=\sum_{a \neq \infty} \delta(a ; g)=1$. Then the relative order and relative lower order of $P_{0}[f]$ with respect to $P_{0}[g]$ are same as those of $f$ with respect to $g$ where $P_{0}[f]$ and $P_{0}[g]$ are homogeneous. i.e.,

$$
\rho_{P_{0}[g]}\left(P_{0}[f]\right)=\rho_{g}(f) \text { and } \lambda_{P_{0}[g]}\left(P_{0}[f]\right)=\lambda_{g}(f) .
$$

Lemma 6. [20] Suppose $f$ be a transcendental meromorphic function of finite order or of non-zero lower order and $\sum_{a \in \mathbb{C} \cup\{\infty\}} \delta_{1}(a ; f)=4$. Also let $g$ be a transcendental entire function of regular growth having non zero finite order and

$\sum_{a \in \mathbb{C} \cup\{\infty\}} \delta_{1}(a ; g)=4$. Then the relative order and relative lower order of $M[f]$ with respect to $M[g]$ are same as those of $f$ with respect to g. i.e.,

$$
\rho_{M[g]}(M[f])=\rho_{g}(f) \text { and } \lambda_{M[g]}(M[f])=\lambda_{g}(f) .
$$

\section{Theorems}

In this section we present the main results of the paper. It is needless to mention that in the paper, the admissibility and homogeneity of $P_{0}[f]$ for meromorphic $f$ will be needed as per the requirements of the theorems.

Theorem 1. Let $g$ be an entire function and $f$ be a meromorphic function either of finite order and non-zero lower order with $\Theta(\infty ; f)=\sum_{a \neq \infty} \delta_{p}(a ; f)=1$ or $\delta(\infty ; f)=\sum_{a \neq \infty} \delta(a ; f)=1$. Also h be an entire function of regular growth having non 
zero finite order with $\Theta(\infty ; h)=\sum_{a \neq \infty} \delta_{p}(a ; h)=1$ or $\delta(\infty ; h)=\sum_{a \neq \infty} \delta(a ; h)=1$ and $0<\lambda_{h}(f) \leq \rho_{h}(f)<\infty$. Then for every positive constant $\mu$ and each $\alpha \in(-\infty, \infty)$,

$$
\liminf _{r \rightarrow \infty} \frac{\left\{\log T_{h}^{-1} T_{f \circ g}(r)\right\}^{1+\alpha}}{\log T_{P_{0}(h)}^{-1} T_{P_{0}(f)}\left(\exp \left(r^{\mu}\right)\right)}=0 \text { if } \mu>\lambda_{g} .
$$

Proof. If $1+\alpha \leq 0$, then the theorem is obvious. We consider $1+\alpha>0$. Since $T_{h}^{-1}(r)$ is an increasing function of $r$, it follows from Lemma 2 for a sequence of values of $r$ tending to infinity that

$$
\begin{aligned}
\log T_{h}^{-1} T_{f \circ g}(r) & <\log T_{h}^{-1} T_{f}\left(\exp \left(r^{\mu}\right)\right) \\
\text { i.e., }, \log T_{h}^{-1} T_{f \circ g}(r) & <\left(\rho_{h}(f)+\varepsilon\right) r^{\mu} .
\end{aligned}
$$

Again for all sufficiently large values of $r$, we get in view of Lemma 5 that

$$
\begin{aligned}
\log T_{P_{0}(h)}^{-1} T_{P_{0}(f)}\left(\exp \left(r^{\mu}\right)\right) & \geq\left(\lambda_{P_{0}(h)}\left(P_{0}(f)\right)-\varepsilon\right) r^{\mu} \\
\text { i.e., } \log T_{P_{0}(h)}^{-1} T_{P_{0}(f)}\left(\exp \left(r^{\mu}\right)\right) & \geq\left(\lambda_{h}(f)-\varepsilon\right) r^{\mu} .
\end{aligned}
$$

Therefore for a sequence of values of $r$ tending to infinity, we obtain from (1) and (2) that

$$
\frac{\left\{\log T_{h}^{-1} T_{f \circ g}(r)\right\}^{1+\alpha}}{\log T_{P_{0}(h)}^{-1} T_{P_{0}(f)}\left(\exp \left(r^{\mu}\right)\right)} \leq \frac{\left(\rho_{h}(f)+\varepsilon\right)^{1+\alpha} r^{\mu(1+\alpha)}}{\left(\lambda_{h}(f)-\varepsilon\right) r^{\mu}} .
$$

So from (3) we obtain that

$$
\liminf _{r \rightarrow \infty} \frac{\left\{\log T_{h}^{-1} T_{f \circ g}(r)\right\}^{1+\alpha}}{\log T_{P_{0}(h)}^{-1} T_{P_{0}(f)}\left(\exp \left(r^{\mu}\right)\right)}=0 .
$$

This proves the theorem.

In the line of Theorem 1 and with the help of Lemma 6, we may state the following theorem without its proof.

Theorem 2. Let $g$ be an entire function and $f$ be a transcendental meromorphic function either of finite order and of non-zero lower order with $\sum_{a \in \mathbb{C} \cup\{\infty\}} \delta_{1}(a ; f)=4$. Also h be a transcendental entire function of regular growth having non zero finite order with $\sum_{a \in \mathbb{C} \cup\{\infty\}} \delta_{1}(a ; h)=4$ and $0<\lambda_{h}(f) \leq \rho_{h}(f)<\infty$. Then for every positive constant $\mu$ and each $\alpha \in(-\infty, \infty)$,

$$
\liminf _{r \rightarrow \infty} \frac{\left\{\log T_{h}^{-1} T_{f \circ g}(r)\right\}^{1+\alpha}}{\log T_{M(h)}^{-1} T_{M(f)}\left(\exp \left(r^{\mu}\right)\right)}=0 \text { if } \mu>\lambda_{g} .
$$

Theorem 3. Let $f$ be a meromorphic function with non zero finite order and lower order, $g$ be an entire function either of finite order or of non-zero lower order such that $\Theta(\infty ; g)=\sum_{a \neq \infty} \delta_{p}(a ; g)=1$ or $\delta(\infty ; g)=\sum_{a \neq \infty} \delta(a ; g)=1$ and $h$ be an entire function of regular growth having non zero finite order and $\Theta(\infty ; h)=\sum_{a \neq \infty} \delta_{p}(a ; h)=1$ or $\delta(\infty ; h)=\sum_{a \neq \infty} \delta(a ; h)=1$. Also let $\rho_{h}(f)<\infty$ and $\lambda_{h}(g)>0$. Then for every positive constant $\mu$ and each $\alpha \in(-\infty, \infty)$,

$$
\liminf _{r \rightarrow \infty} \frac{\left\{\log T_{h}^{-1} T_{f \circ g}(r)\right\}^{1+\alpha}}{\log T_{P_{0}(h)}^{-1} T_{P_{0}(g)}\left(\exp \left(r^{\mu}\right)\right)}=0 \text { if } \mu>\lambda_{g} .
$$

The proof is omitted as it can be carried out in the line of Theorem 1. 
Theorem 4. Let $f$ be a meromorphic function with non zero finite order and lower order, $g$ be a transcendental entire function either of finite order or of non-zero lower order such that $\sum_{a \in \mathbb{C} \cup\{\infty\}} \delta_{1}(a ; g)=4$ and h be a transcendental entire function of regular growth having non zero finite order and $\sum_{a \in \mathbb{C} \cup\{\infty\}} \delta_{1}(a ; h)=4$. Also let $\rho_{h}(f)<\infty$ and $\lambda_{h}(g)>0$. Then for every positive constant $\mu$ and each $\alpha \in(-\infty, \infty)$,

$$
\liminf _{r \rightarrow \infty} \frac{\left\{\log T_{h}^{-1} T_{f \circ g}(r)\right\}^{1+\alpha}}{\log T_{M(h)}^{-1} T_{M(g)}\left(\exp \left(r^{\mu}\right)\right)}=0 \text { if } \mu>\lambda_{g} .
$$

The proof of the above theorem is omitted as it can be carried out in the line of Theorem 3 and with the help of Lemma 6.

Theorem 5. Let $f$ be a meromorphic function of finite order with $\Theta(\infty ; f)=\sum_{a \neq \infty} \delta_{p}(a ; f)=1$ or $\delta(\infty ; f)=\sum_{a \neq \infty} \delta(a ; f)=1$, $g$ be an entire function with non zero finite lower order and $h$ be an entire function of regular growth having non zero finite order with $\Theta(\infty ; h)=\sum_{a \neq \infty} \delta_{p}(a ; h)=1$ or $\delta(\infty ; h)=\sum_{a \neq \infty} \delta(a ; h)=1$. Also let $\lambda_{h}(f)>0$ and $\rho_{h}(g)<\infty$. Then for every positive constant $\mu$ and each $\alpha \in(-\infty, \infty)$,

$$
\liminf _{r \rightarrow \infty} \frac{\left\{\log T_{h}^{-1} T_{f \circ g}(r)\right\}^{1+\alpha}}{\log T_{P_{0}(h)}^{-1} T_{P_{0}(f)}\left(\exp \left(r^{\mu}\right)\right)}=0 \text { if } \mu>\lambda_{g} .
$$

Theorem 6. Let $f$ be a meromorphic function with finite order, $g$ be an entire function non zero finite lower order with $\Theta(\infty ; g)=\sum_{a \neq \infty} \delta_{p}(a ; g)=1$ or $\delta(\infty ; g)=\sum_{a \neq \infty} \delta(a ; g)=1$ and he an entire function of regular growth having non zero finite order with $\Theta(\infty ; h)=\sum_{a \neq \infty} \delta_{p}(a ; h)=1$ or $\delta(\infty ; h)=\sum_{a \neq \infty} \delta(a ; h)=1$. Also let $0<\lambda_{h}(g) \leq \rho_{h}(g)<\infty$. Then for every positive constant $\mu$ and each $\alpha \in(-\infty, \infty)$,

$$
\liminf _{r \rightarrow \infty} \frac{\left\{\log T_{h}^{-1} T_{f \circ g}(r)\right\}^{1+\alpha}}{\log T_{P_{0}(h)}^{-1} T_{P_{0}(g)}\left(\exp \left(r^{\mu}\right)\right)}=0 \text { if } \mu>\lambda_{g} .
$$

We omit the proofs of Theorem 5 and Theorem 6 as those can be carried out in the line of Theorem 1 and Theorem 3 respectively and with the help of Lemma 3.

In the line of Theorem 5 and Theorem 6 and with the help of Lemma 6 we may state the following two theorems without their proofs.

Theorem 7. Let $f$ be a transcendental meromorphic function of finite order with $\sum_{a \in \mathbb{C} \cup\{\infty\}} \delta_{1}(a ; f)=4$, g be an entire function with non zero finite lower order and $h$ be a transcendental entire function of regular growth having non zero finite order with $\sum_{a \in \mathbb{C} \cup\{\infty\}} \delta_{1}(a ; h)=4$. Also let $\lambda_{h}(f)>0$ and $\rho_{h}(g)<\infty$. Then for every positive constant $\mu$ and each $\alpha \in(-\infty, \infty)$,

$$
\liminf _{r \rightarrow \infty} \frac{\left\{\log T_{h}^{-1} T_{f \circ g}(r)\right\}^{1+\alpha}}{\log T_{M(h)}^{-1} T_{M(f)}\left(\exp \left(r^{\mu}\right)\right)}=0 \text { if } \mu>\lambda_{g}
$$

Theorem 8. Let $f$ be a meromorphic function with finite order, $g$ be a transcendental entire function non zero finite lower order with $\sum_{a \in \mathbb{C} \cup\{\infty\}} \delta_{1}(a ; g)=4$ and $h$ be a transcendental entire function of regular growth having non zero finite order 
with $\sum_{a \in \mathbb{C} \cup\{\infty\}} \delta_{1}(a ; h)=4$. Also let $0<\lambda_{h}(g) \leq \rho_{h}(g)<\infty$. Then for every positive constant $\mu$ and each $\alpha \in(-\infty, \infty)$,

$$
\liminf _{r \rightarrow \infty} \frac{\left\{\log T_{h}^{-1} T_{f \circ g}(r)\right\}^{1+\alpha}}{\log T_{M(h)}^{-1} T_{M(g)}\left(\exp \left(r^{\mu}\right)\right)}=0 \text { if } \mu>\lambda_{g} .
$$

Theorem 9. Suppose $f$ be a meromorphic function either of finite order or of non-zero lower order such that $\Theta(\infty ; f)=$ $\sum_{a \neq \infty} \delta_{p}(a ; f)=1$ or $\delta(\infty ; f)=\sum_{a \neq \infty} \delta(a ; f)=1$. Also let $h$ be an entire function of regular growth having non zero finite order with $\Theta(\infty ; h)=\sum_{a \neq \infty} \delta_{p}(a ; h)=1$ or $\delta(\infty ; h)=\sum_{a \neq \infty} \delta(a ; h)=1$ and $g$ be any entire function such that $\rho_{h}(f)<\infty$ and $\lambda_{h}(f \circ g)=\infty$. Then

$$
\lim _{r \rightarrow \infty} \frac{\log T_{h}^{-1} T_{f \circ g}(r)}{\log T_{P_{0}[h]}^{-1} T_{P_{0}[f]}(r)}=\infty
$$

Proof. Let us suppose that the conclusion of the theorem do not hold. Then we can find a constant $\beta>0$ such that for a sequence of values of $r$ tending to infinity,

$$
\log T_{h}^{-1} T_{f \circ g}(r) \leq \beta \log T_{P_{0}[h]}^{-1} T_{P_{0}[f]}(r)
$$

Again it follows for all sufficiently large values of $r$ and in view of Lemma 5 that

$$
\begin{aligned}
\log T_{P_{0}[h]}^{-1} T_{P_{0}[f]}(r) & \leq\left(\rho_{P_{0}[h]}\left(P_{0}[f]\right)+\varepsilon\right) \log r \\
\text { i.e., }, \log T_{P_{0}[h]}^{-1} T_{P_{0}[f]}(r) & \leq\left(\rho_{h}(f)+\varepsilon\right) \log r .
\end{aligned}
$$

Thus from (4) and (5), we have for a sequence of values of $r$ tending to infinity that

$$
\begin{aligned}
\log T_{h}^{-1} T_{f \circ g}(r) & \leq \beta\left(\rho_{h}(f)+\varepsilon\right) \log r \\
\text { i.e., } \frac{\log T_{h}^{-1} T_{f \circ g}(r)}{\log r} \leq \frac{\beta\left(\rho_{h}(f)+\varepsilon\right) \log r}{\log r} & \leq \lambda_{h}(f \circ g)<\infty .
\end{aligned}
$$

This is a contradiction. Hence the theorem follows.

Remark. Theorem 9 is also valid with "limit superior" instead of "limit" if $\lambda_{h}(f \circ g)=\infty$ is replaced by $\rho_{h}(f \circ g)=\infty$ and the other conditions remain the same.

Corollary 1. Under the assumptions of Theorem 9 and Remark 3,

$$
\lim _{r \rightarrow \infty} \frac{T_{h}^{-1} T_{f \circ g}(r)}{T_{P_{0}[h]}^{-1} T_{P_{0}[f]}(r)}=\infty \text { and } \limsup _{r \rightarrow \infty} \frac{T_{h}^{-1} T_{f \circ g}(r)}{T_{P_{0}[h]}^{-1} T_{P_{0}[f]}(r)}=\infty
$$

respectively hold.

The proof is omitted.

Analogously one may also state the following theorem and corollaries without their proofs as those may be carried out in the line of Remark 3, Theorem 9 and Corollary 1 respectively. 
Theorem 10. Let $g$ be an entire function either of finite order or of non-zero lower order such that $\Theta(\infty ; g)=\sum_{a \neq \infty} \delta_{p}(a ; g)=1$ or $\delta(\infty ; g)=\sum_{a \neq \infty} \delta(a ; g)=1$. Also let $h$ be an entire function of regular growth having non zero finite order with $\Theta(\infty ; h)=\sum_{a \neq \infty} \delta_{p}(a ; h)=1$ or $\delta(\infty ; h)=\sum_{a \neq \infty} \delta(a ; h)=1$ and $f$ be any meromorphic function such that $\rho_{h}(g)<\infty$ and $\rho_{h}(f \circ g)=\infty$. Then

$$
\limsup _{r \rightarrow \infty} \frac{\log T_{h}^{-1} T_{f \circ g}(r)}{\log T_{P_{0}[h]}^{-1} T_{P_{0}[g]}(r)}=\infty
$$

Remark. Theorem 10 is also valid with "limit" instead of "limit superior" if $\rho_{h}(f \circ g)=\infty$ is replaced by $\lambda_{h}(f \circ g)=\infty$ and the other conditions remain the same.

Corollary 2. Under the assumptions of Theorem 10 and Remark 3,

$$
\limsup _{r \rightarrow \infty} \frac{T_{h}^{-1} T_{f \circ g}(r)}{T_{P_{0}[h]}^{-1} T_{P_{0}[g]}(r)}=\infty \text { and } \lim _{r \rightarrow \infty} \frac{T_{h}^{-1} T_{f \circ g}(r)}{T_{P_{0}[h]}^{-1} T_{P_{0}[g]}(r)}=\infty
$$

respectively hold.

In the line of Theorem 9 and Theorem 10 and with the help of Lemma 6, we may state the following two theorems without their proofs.

Theorem 11. Suppose $f$ be a transcendental meromorphic function either of finite order or of non-zero lower order such that $\sum_{a \in \mathbb{C} \cup\{\infty\}} \delta_{1}(a ; f)=4$. Also let $h$ be a transcendental entire function of regular growth having non zero finite order with $\sum_{a \in \mathbb{C} \cup\{\infty\}} \delta_{1}(a ; h)=4$ and $g$ be any entire function such that $\rho_{h}(f)<\infty$ and $\lambda_{h}(f \circ g)=\infty$. Then

$$
\lim _{r \rightarrow \infty} \frac{\log T_{h}^{-1} T_{f \circ g}(r)}{\log T_{M[h]}^{-1} T_{M[f]}(r)}=\infty
$$

Remark. Theorem 11 is also valid with "limit superior" instead of "limit" if $\lambda_{h}(f \circ g)=\infty$ is replaced by $\rho_{h}(f \circ g)=\infty$ and the other conditions remain the same.

Corollary 3. Under the assumptions of Theorem 11 and Remark 3,

$$
\lim _{r \rightarrow \infty} \frac{T_{h}^{-1} T_{f \circ g}(r)}{T_{M[h]}^{-1} T_{M[f]}(r)}=\infty \text { and } \limsup _{r \rightarrow \infty} \frac{T_{h}^{-1} T_{f \circ g}(r)}{T_{M[h]}^{-1} T_{M[f]}(r)}=\infty
$$

respectively hold.

The proof is omitted.

Theorem 12. Let $g$ be a transcendental entire function either of finite order or of non-zero lower order such that $\sum_{a \in \mathbb{C} \cup\{\infty\}} \delta_{1}(a ; g)=4$. Also let $h$ be a transcendental entire function of regular growth having non zero finite order with $\sum_{a \in \mathbb{C} \cup\{\infty\}} \delta_{1}(a ; h)=4$ and $f$ be any meromorphic function such that $\rho_{h}(g)<\infty$ and $\rho_{h}(f \circ g)=\infty$.Then

$$
\limsup _{r \rightarrow \infty} \frac{\log T_{h}^{-1} T_{f \circ g}(r)}{\log T_{M[h]}^{-1} T_{M[g]}(r)}=\infty
$$


Remark. Theorem 12 is also valid with "limit" instead of "limit superior" if $\rho_{h}(f \circ g)=\infty$ is replaced by $\lambda_{h}(f \circ g)=\infty$ and the other conditions remain the same.

Corollary 4. Under the assumptions of Theorem 12 and Remark 3,

$$
\limsup _{r \rightarrow \infty} \frac{T_{h}^{-1} T_{f \circ g}(r)}{T_{M[h]}^{-1} T_{M[g]}(r)}=\infty \text { and } \lim _{r \rightarrow \infty} \frac{T_{h}^{-1} T_{f \circ g}(r)}{T_{M[h]}^{-1} T_{M[g]}(r)}=\infty
$$

respectively hold.

Theorem 13. Let $f$ be a meromorphic function either of finite order or of non-zero lower order with $\Theta(\infty ; f)=\sum_{a \neq \infty} \delta_{p}(a ; f)=1$ or $\delta(\infty ; f)=\sum_{a \neq \infty} \delta(a ; f)=1, g$ be an entire function and $h$ be an entire function of regular growth having non zero finite order with $\Theta(\infty ; h)=\sum_{a \neq \infty} \delta_{p}(a ; h)=1$ or $\delta(\infty ; h)=\sum_{a \neq \infty} \delta(a ; h)=1$ and satisfy the Property (A). Also let $\lambda_{g}<\lambda_{h}(f) \leq \rho_{h}(f)<\infty$. Then

$$
\liminf _{r \rightarrow \infty} \frac{\log T_{h}^{-1} T_{f \circ g}(r)}{T_{P_{0}[h]}^{-1} T_{P_{0}[f]}(r)}=0
$$

Proof. Let $\beta>2$ and $\delta>1$. Since $T_{h}^{-1}(r)$ is an increasing function of $r$, it follows from Lemma 1 and Lemma 4 , for all sufficiently large values of $r$ that

$$
\begin{aligned}
T_{h}^{-1} T_{f \circ g}(r) & \leqslant T_{h}^{-1}\left[\{1+o(1)\} T_{f}\left(M_{g}(r)\right)\right] \\
\text { i.e., } T_{h}^{-1} T_{f \circ g}(r) & \leqslant \beta\left[T_{h}^{-1} T_{f}\left(M_{g}(r)\right)\right]^{\delta} \\
\text { i.e., } \log T_{h}^{-1} T_{f \circ g}(r) & \leqslant \delta \log T_{h}^{-1} T_{f}\left(M_{g}(r)\right)+O(1) .
\end{aligned}
$$

Therefore from above, we get for a sequence of values of $r$ tending to infinity that

$$
\begin{gathered}
\log T_{h}^{-1} T_{f \circ g}(r) \leq \delta\left(\rho_{h}(f)+\varepsilon\right) \log M_{g}(r)+O(1) \\
\text { i.e., } \log T_{h}^{-1} T_{f \circ g}(r) \leq \delta\left(\rho_{h}(f)+\varepsilon\right) r^{\lambda_{g}+\varepsilon}+O(1) .
\end{gathered}
$$

Again from the definition of relative order, we obtain in view of Lemma 5 for all sufficiently large values of $r$ that

$$
\begin{aligned}
T_{P_{0}[h]}^{-1} T_{P_{0}[f]}(r) & \geqslant r^{\left(\lambda_{P_{0}(h)}\left(P_{0}(f)\right)-\varepsilon\right)} \\
\text { i.e., } T_{P_{0}[h]}^{-1} T_{P_{0}[f]}(r) & \geqslant r^{\left(\lambda_{h}(f)-\varepsilon\right)} .
\end{aligned}
$$

Thus in view of (7) and (8), we get for a sequence of values of $r$ tending to infinity,

$$
\frac{\log T_{h}^{-1} T_{f \circ g}(r)}{T_{P_{0}[h]}^{-1} T_{P_{0}[f]}(r)}<\frac{\delta\left(\rho_{h}(f)+\varepsilon\right) r^{\lambda_{g}+\varepsilon}+O(1)}{r^{\left(\lambda_{h}(f)-\varepsilon\right)}} .
$$

Now as $\lambda_{g}<\lambda_{h}(f)$, we can choose $\varepsilon(>0)$ in such a way that $\lambda_{g}+\varepsilon<\lambda_{h}(f)-\varepsilon$ and the theorem follows from (9).

Remark. If we take $\rho_{g}<\lambda_{h}(f) \leq \rho_{h}(f)<\infty$ instead of $\lambda_{g}<\lambda_{h}(f) \leq \rho_{h}(f)<\infty$ and the other conditions remain the same, the conclusion of Theorem 13 remains valid with "limit inferior" replaced by "limit". 
Theorem 14. Let $f$ be a transcendental meromorphic function either of finite order or of non-zero lower order with $\sum_{a \in \mathbb{C} \cup\{\infty\}} \delta_{1}(a ; f)=4, g$ be an entire function and $h$ be a transcendental entire function of regular growth having non zero finite order with $\sum_{a \in \mathbb{C} \cup\{\infty\}} \delta_{1}(a ; h)=4$ and satisfy the Property $(A)$. Also let $\lambda_{g}<\lambda_{h}(f) \leq \rho_{h}(f)<\infty$. Then

$$
\liminf _{r \rightarrow \infty} \frac{\log T_{h}^{-1} T_{f \circ g}(r)}{T_{M[h]}^{-1} T_{M[f]}(r)}=0
$$

The proof of the above theorem is omitted as it can be carried out in the line of Theorem 13 and with the help of Lemma 6.

Remark. If we consider $\rho_{g}<\lambda_{h}(f) \leq \rho_{h}(f)<\infty$ instead of $\lambda_{g}<\lambda_{h}(f) \leq \rho_{h}(f)<\infty$ and the other conditions remain the same, the conclusion of Theorem 14 remains valid with "limit inferior" replaced by "limit".

Theorem 15. Let $f$ be a meromorphic function, $g$ be an entire function either of finite order or of non-zero lower order with $\Theta(\infty ; f)=\sum_{a \neq \infty} \delta_{p}(a ; f)=1$ or $\delta(\infty ; f)=\sum_{a \neq \infty} \delta(a ; f)=1$ and h be an entire function of regular growth having non zero finite order with $\Theta(\infty ; h)=\sum_{a \neq \infty} \delta_{p}(a ; h)=1$ or $\delta(\infty ; h)=\sum_{a \neq \infty} \delta(a ; h)=1$. Also let $\rho_{h}(f \circ g)<\infty$ and $\lambda_{h}(g)>0$. Then

$$
\lim _{r \rightarrow \infty} \frac{\left[\log T_{h}^{-1} T_{f \circ g}(r)\right]^{2}}{T_{P_{0}(h)}^{-1} T_{P_{0}(g)}(\exp r) \cdot \log T_{P_{0}(h)}^{-1} T_{P_{0}(g)}(r)}=0 .
$$

Proof. For any arbitrary positive $\varepsilon$, we have in view of Lemma 5 for all sufficiently large values of $r$ that

$$
\log T_{h}^{-1} T_{f \circ g}(r) \leq\left(\rho_{h}(f \circ g)+\varepsilon\right) \log r
$$

and

$$
\begin{aligned}
\log T_{P_{0}(h)}^{-1} T_{P_{0}(g)}(r) & \geq\left(\lambda_{P_{0}(h)}\left(P_{0}(g)\right)-\varepsilon\right) \log r \\
\text { i.e., } \log T_{P_{0}(h)}^{-1} T_{P_{0}(g)}(r) & \geq\left(\lambda_{h}(g)-\varepsilon\right) \log r .
\end{aligned}
$$

Similarly, for all sufficiently large values of $r$ we have

$$
\begin{gathered}
\log T_{P_{0}(h)}^{-1} T_{P_{0}(g)}(\exp r) \geq\left(\lambda_{L(h)}(L(g))-\varepsilon\right) r \\
\text { i.e., } T_{P_{0}(h)}^{-1} T_{P_{0}(g)}(\exp r) \geq \exp \left[\left(\lambda_{h}(g)-\varepsilon\right) r\right] .
\end{gathered}
$$

From (10) and (11), we have for all sufficiently large values of $r$ that

$$
\frac{\log T_{h}^{-1} T_{f \circ g}(r)}{\log T_{P_{0}(h)}^{-1} T_{P_{0}(g)}(r)} \leq \frac{\left(\rho_{h}(f \circ g)+\varepsilon\right) \log r}{\left(\lambda_{h}(g)-\varepsilon\right) \log r} .
$$

As $\varepsilon(>0)$ is arbitrary, we obtain from above that

$$
\limsup _{r \rightarrow \infty} \frac{\log T_{h}^{-1} T_{f \circ g}(r)}{\log T_{P_{0}(h)}^{-1} T_{P_{0}(g)}(r)} \leq \frac{\rho_{h}(f \circ g)}{\lambda_{h}(g)} .
$$


Again from (10) and (12), we get for all sufficiently large values of $r$ that

$$
\frac{\log T_{h}^{-1} T_{f \circ g}(r)}{T_{P_{0}(h)}^{-1} T_{P_{0}(g)}(\exp r)} \leq \frac{\left(\rho_{h}(f \circ g)+\varepsilon\right) \log r}{\exp \left[\left(\lambda_{h}(g)-\varepsilon\right) r\right]} .
$$

Since $\varepsilon(>0)$ is arbitrary, it follows from above that

$$
\begin{gathered}
\limsup _{r \rightarrow \infty} \frac{\log T_{h}^{-1} T_{f \circ g}(r)}{T_{P_{0}(h)}^{-1} T_{P_{0}(g)}(\exp r)}=0 \\
\text { i.e., } \lim _{r \rightarrow \infty} \frac{\log T_{h}^{-1} T_{f \circ g}(r)}{T_{P_{0}(h)}^{-1} T_{P_{0}(g)}(\exp r)}=0 .
\end{gathered}
$$

Thus the theorem follows from (13) and (14).

In view of Theorem 15, the following two theorems can be carried out. Hence their proofs are omitted.

Theorem 16. Let $f$ a meromorphic function either of finite order or of non-zero lower order such that $\Theta(\infty ; f)=\sum_{a \neq \infty} \delta_{p}(a ; f)=1$ or $\delta(\infty ; f)=\sum_{a \neq \infty} \delta(a ; f)=1$, g be any entire function and $h$ be an entire function of regular growth having non zero finite order with $\Theta(\infty ; h)=\sum_{a \neq \infty} \delta_{p}(a ; h)=1$ or $\delta(\infty ; h)=\sum_{a \neq \infty} \delta(a ; h)=1$. Also let $\rho_{h}(f \circ g)<\infty$ and $\lambda_{h}(f)>0$. Then

$$
\lim _{r \rightarrow \infty} \frac{\left[\log T_{h}^{-1} T_{f \circ g}(r)\right]^{2}}{T_{P_{0}(h)}^{-1} T_{P_{0}(f)}(\exp (r)) \cdot \log T_{P_{0}(h)}^{-1} T_{P_{0}(f)}(r)}=0 .
$$

Theorem 17. Let $f$ be a meromorphic function either of finite order or of non-zero lower order such that $\Theta(\infty ; f)=$ $\sum_{a \neq \infty} \delta_{p}(a ; f)=1$ or $\delta(\infty ; f)=\sum_{a \neq \infty} \delta(a ; f)=1, g$ be an entire function either of finite order or of non-zero lower order such that $\sum_{a \neq \infty} \Theta(a ; g)=2$ and $h$ be an entire function of regular growth having non zero finite order and $\Theta(\infty ; h)=$ $\sum_{a \neq \infty} \delta_{p}(a ; h)=1$ or $\delta(\infty ; h)=\sum_{a \neq \infty} \delta(a ; h)=1$. Also let $\rho_{h}(f \circ g)<\infty, \lambda_{h}(g)>0$ and $\lambda_{h}(f)>0$. Then

$$
\begin{aligned}
& \lim _{r \rightarrow \infty} \frac{\left[\log T_{h}^{-1} T_{f \circ g}(r)\right]^{2}}{T_{P_{0}(h)}^{-1} T_{P_{0}(g)}(\exp r) \cdot \log T_{P_{0}(h)}^{-1} T_{P_{0}(f)}(r)}=0 \quad \text { and } \\
& \lim _{r \rightarrow \infty} \frac{\left[\log T_{h}^{-1} T_{f \circ g}(r)\right]^{2}}{T_{P_{0}(h)}^{-1} T_{P_{0}(f)}(\exp r) \cdot \log T_{P_{0}(h)}^{-1} T_{P_{0}(g)}(r)}=0 .
\end{aligned}
$$

In the line of Theorem 15, Theorem 16 and Theorem 17 and with the help of Lemma 6 we may state the following three theorems without their proofs :

Theorem 18. Let $f$ be a meromorphic function, $g$ be a transcendental entire function either of finite order or of non-zero lower order with $\sum_{a \in \mathbb{C} \cup\{\infty\}} \delta_{1}(a ; g)=4$ and $h$ be a transcendental entire function of regular growth having non zero finite order with $\sum_{a \in \mathbb{C} \cup\{\infty\}} \delta_{1}(a ; h)=4$. Also let $\rho_{h}(f \circ g)<\infty$ and $\lambda_{h}(g)>0$. Then

$$
\lim _{r \rightarrow \infty} \frac{\left[\log T_{h}^{-1} T_{f \circ g}(r)\right]^{2}}{T_{M(h)}^{-1} T_{M(g)}(\exp r) \cdot \log T_{M(h)}^{-1} T_{M(g)}(r)}=0 .
$$

Theorem 19. Let $f$ a transcendental meromorphic function either of finite order or of non-zero lower order such that $\sum_{a \in \mathbb{C} \cup\{\infty\}} \delta_{1}(a ; f)=4, g$ be any entire function and $h$ be a transcendental entire function of regular growth having non zero 
finite order with $\sum_{a \in \mathbb{C} \cup\{\infty\}} \delta_{1}(a ; h)=4$. Also let $\rho_{h}(f \circ g)<\infty$ and $\lambda_{h}(f)>0$. Then

$$
\lim _{r \rightarrow \infty} \frac{\left[\log T_{h}^{-1} T_{f \circ g}(r)\right]^{2}}{T_{M(h)}^{-1} T_{M(f)}(\exp r) \cdot \log T_{M(h)}^{-1} T_{M(f)}(r)}=0
$$

Theorem 20. Let $f$ be a transcendental meromorphic function either of finite order or of non-zero lower order such that $\sum_{a \in \mathbb{C} \cup\{\infty\}} \delta_{1}(a ; f)=4, g$ be a transcendental entire function either of finite order or of non-zero lower order such that $\sum_{a \in \mathbb{C} \cup\{\infty\}} \delta_{1}(a ; g)=4$ and $h$ be a transcendental entire function of regular growth having non zero finite order and $\sum_{a \in \mathbb{C} \cup\{\infty\}} \delta_{1}(a ; h)=4$. Also let $\rho_{h}(f \circ g)<\infty, \lambda_{h}(g)>0$ and $\lambda_{h}(f)>0$. Then

$$
\begin{aligned}
& \lim _{r \rightarrow \infty} \frac{\left[\log T_{h}^{-1} T_{f \circ g}(r)\right]^{2}}{T_{M(h)}^{-1} T_{M(g)}(\exp r) \cdot \log T_{M(h)}^{-1} T_{M(f)}(r)}=0 \quad \text { and } \\
& \lim _{r \rightarrow \infty} \frac{\left[\log T_{h}^{-1} T_{f \circ g}(r)\right]^{2}}{T_{M(h)}^{-1} T_{M(f)}(\exp r) \cdot \log T_{M(h)}^{-1} T_{M(g)}(r)}=0 .
\end{aligned}
$$

Theorem 21. Let $f$ be a meromorphic function either of finite order or of non-zero lower order with $\Theta(\infty ; f)=\sum_{a \neq \infty} \delta_{p}(a ; f)=1$ or $\delta(\infty ; f)=\sum_{a \neq \infty} \delta(a ; f)=1, g$ be an entire function with finite order and $h$ be an entire function of regular growth having non zero finite order with $\Theta(\infty ; h)=\sum_{a \neq \infty} \delta_{p}(a ; h)=1$ or $\delta(\infty ; h)=\sum_{a \neq \infty} \delta(a ; h)=1$ and satisfy the Property (A). Also let $0<\lambda_{h}(f) \leq \rho_{h}(f)<\infty$. Then

$$
\limsup _{r \rightarrow \infty} \frac{\log ^{[2]} T_{h}^{-1} T_{f \circ g}(r)}{\log T_{P_{0}(h)}^{-1} T_{P_{0}(f)}(r)} \leq \frac{\rho_{g}}{\lambda_{h}(f)} .
$$

Proof. From (6) and in view of Lemma 5, it follows for all sufficiently large values of $r$ that

$$
\begin{aligned}
& \log ^{[2]} T_{h}^{-1} T_{f \circ g}(r) \leq \log ^{[2]} M_{g}(r)+O(1) \\
& \text { i.e., } \frac{\log ^{[2]} T_{h}^{-1} T_{f \circ g}(r)}{\log T_{P_{0}(h)}^{-1} T_{P_{0}(f)}(r)} \leq \frac{\log ^{[2]} M_{g}(r)+O(1)}{\log r} \cdot \frac{\log r}{\log T_{P_{0}(h)}^{-1} T_{P_{0}(f)}(r)} \\
& \text { i.e., } \limsup _{r \rightarrow \infty} \frac{\log { }^{[2]} T_{h}^{-1} T_{f \circ g}(r)}{\log T_{P_{0}(h)}^{-1} T_{P_{0}(f)}(r)} \leq \limsup _{r \rightarrow \infty} \frac{\log M_{g}^{[2]}(r)+O(1)}{\log r} \cdot \limsup _{r \rightarrow \infty} \frac{\log r}{\log T_{P_{0}(h)}^{-1} T_{P_{0}(f)}(r)} \\
& \text { i.e., } \limsup _{r \rightarrow \infty} \frac{\log T_{h}^{[2]} T_{f \circ g}(r)}{\log T_{P_{0}(h)}^{-1} T_{P_{0}(f)}(r)} \leq \rho_{g} \cdot \frac{1}{\lambda_{P_{0}(h)}\left(P_{0}(f)\right)}=\frac{\rho_{g}}{\lambda_{h}(f)} .
\end{aligned}
$$

This proves the theorem.

Theorem 22. Let $f$ be a meromorphic function, $g$ be an entire function of finite order with $\Theta(\infty ; g)=\sum_{a \neq \infty} \delta_{p}(a ; g)=1$ or $\delta(\infty ; g)=\sum_{a \neq \infty} \delta(a ; g)=1$ and $h$ be an entire function of regular growth having non zero finite order with $\Theta(\infty ; h)=$ $\sum_{a \neq \infty} \delta_{p}(a ; h)=1$ or $\delta(\infty ; h)=\sum_{a \neq \infty} \delta(a ; h)=1$ and satisfy the Property $(A)$. Also let $\rho_{h}(f)<\infty$ and $\lambda_{h}(g)>0$. Then

$$
\limsup _{r \rightarrow \infty} \frac{\log { }^{[2]} T_{h}^{-1} T_{f \circ g}(r)}{\log T_{P_{0}(h)}^{-1} T_{P_{0}(g)}(r)} \leq \frac{\rho_{g}}{\lambda_{h}(g)} .
$$

The proof of Theorem 22 is omitted as it can be carried out in the line of Theorem 21. 
Theorem 23.Let $f$ be a transcendental meromorphic function either of finite order or of non-zero lower order with $\sum_{a \in \mathbb{C} \cup\{\infty\}} \delta_{1}(a ; f)=4, g$ be an entire function with finite order and $h$ be a transcendental entire function of regular growth having non zero finite order with $\sum_{a \in \mathbb{C} \cup\{\infty\}} \delta_{1}(a ; h)=4$ and satisfy the Property $(A)$. Also let $0<\lambda_{h}(f) \leq \rho_{h}(f)<\infty$. Then

$$
\limsup _{r \rightarrow \infty} \frac{\log ^{[2]} T_{h}^{-1} T_{f \circ g}(r)}{\log T_{M(h)}^{-1} T_{M(f)}(r)} \leq \frac{\rho_{g}}{\lambda_{h}(f)} .
$$

Theorem 24. Let $f$ be a meromorphic function, $g$ be a transcendental entire function of finite order with $\sum_{a \in \mathbb{C} \cup\{\infty\}} \delta_{1}(a ; g)=$ 4 and $h$ be a transcendental entire function of regular growth having non zero finite order with $\sum_{a \in \mathbb{C} \cup\{\infty\}} \delta_{1}(a ; h)=4$ and satisfy the Property (A). Also let $\rho_{h}(f)<\infty$ and $\lambda_{h}(g)>0$. Then

$$
\limsup _{r \rightarrow \infty} \frac{\log ^{[2]} T_{h}^{-1} T_{f \circ g}(r)}{\log T_{M(h)}^{-1} T_{M(g)}(r)} \leq \frac{\rho_{g}}{\lambda_{h}(g)} .
$$

The proof of the above two theorems are omitted as those can be carried out in the line of Theorem 21 and Theorem 22 respectively and with the help of Lemma 6.

Theorem 25. Let $f$ be a meromorphic function either of finite order or of non-zero lower order such that $\Theta(\infty ; f)=$ $\sum_{a \neq \infty} \delta_{p}(a ; f)=1$ or $\delta(\infty ; f)=\sum_{a \neq \infty} \delta(a ; f)=1, g$ be an entire function with finite order and $h$ be an entire function of regular growth having non zero finite order with $\Theta(\infty ; h)=\sum_{a \neq \infty} \delta_{p}(a ; h)=1$ or $\delta(\infty ; h)=\sum_{a \neq \infty} \delta(a ; h)=1$ and satisfy the Property (A). Also let $0<\lambda_{h}(f) \leq \rho_{h}(f)<\infty$. Then

$$
\lim _{r \rightarrow \infty} \frac{\log T_{h}^{-1} T_{f \circ g}(r)}{\log T_{P_{0}(h)}^{-1} T_{P_{0}(f)}\left(\exp r^{\mu}\right)}=\infty
$$

where $\rho_{g}<\mu<\infty$.

Proof. Let us consider $\beta>2$ and $\delta>1$. As $T_{h}^{-1}(r)$ is an increasing function of $r$, in view of Lemma 1 we get from(6) for all sufficiently large values of $r$,

$$
\log T_{h}^{-1} T_{f \circ g}(r) \leq \delta\left(\rho_{h}(f)+\varepsilon\right) r^{\rho_{g}+\varepsilon}+O(1) .
$$

Also from the definition of the relative lower order and in view of Lemma 5, we obtain for all sufficiently large values of $r$ that

$$
\begin{aligned}
\log T_{P_{0}(h)}^{-1} T_{P_{0}(f)}\left(\exp \left(r^{\mu}\right)\right) & \geq\left(\lambda_{P_{0}(h)}\left(P_{0}(f)\right)-\varepsilon\right) \log \left\{\exp \left(r^{\mu}\right)\right\} \\
\text { i.e., } \log T_{P_{0}(h)}^{-1} T_{P_{0}(f)}\left(\exp r^{\mu}\right) & \geq\left(\lambda_{h}(f)-\varepsilon\right) r^{\mu} .
\end{aligned}
$$

Now from (15) and (16), it follows for all sufficiently large values of $r$ that

$$
\frac{\log T_{h}^{-1} T_{f \circ g}(r)}{\log T_{P_{0}(h)}^{-1} T_{P_{0}(f)}\left(\exp r^{\mu}\right)} \leq \frac{\delta\left(\rho_{h}(f)+\varepsilon\right) r^{\rho_{g}+\varepsilon}+O(1)}{\left(\lambda_{h}(f)-\varepsilon\right) r^{\mu}}
$$

As $\rho_{g}<\mu$, we can choose $\varepsilon(>0)$ in such a way that

$$
\rho_{g}+\varepsilon<\mu
$$


Thus from (17) and (18), we obtain that

$$
\lim _{r \rightarrow \infty} \frac{\log T_{h}^{-1} T_{f \circ g}(r)}{\log T_{P_{0}(h)}^{-1} T_{P_{0}(f)}\left(\exp r^{\mu}\right)}=0 .
$$

Thus the theorem follows.

In the line of Theorem 25, we may state the following theorem without its proof:

Theorem 26. Let $f$ be a meromorphic function, $g$ be an entire function either of finite order or of non-zero lower order with $\Theta(\infty ; g)=\sum_{a \neq \infty} \delta_{p}(a ; g)=1$ or $\delta(\infty ; g)=\sum_{a \neq \infty} \delta(a ; g)=1$ and $h$ be an entire function of regular growth having non zero finite order with $\Theta(\infty ; h)=\sum_{a \neq \infty} \delta_{p}(a ; h)=1$ or $\delta(\infty ; h)=\sum_{a \neq \infty} \delta(a ; h)=1$ and satisfy the Property (A). Also let $\lambda_{h}(g)>0$ and $\rho_{h}(f)<\infty$. Then for every $\mu$ with $\rho_{g}<\mu<\infty$,

$$
\lim _{r \rightarrow \infty} \frac{\log T_{h}^{-1} T_{f \circ g}(r)}{\log T_{P_{0}(h)}^{-1} T_{P_{0}(g)}\left(\exp r^{\mu}\right)}=0 .
$$

In the line of Theorem 25 and Theorem 26 and with the help of Lemma 6, we may state the following two theorems without their proofs :

Theorem 27. Let $f$ be a transcendental meromorphic function either of finite order or of non-zero lower order such that $\sum_{a \in \mathbb{C} \cup\{\infty\}} \delta_{1}(a ; f)=4, g$ be an entire function with finite order and $h$ be a transcendental entire function of regular growth having non zero finite order with $\sum_{a \in \mathbb{C} \cup\{\infty\}} \delta_{1}(a ; h)=4$ and satisfy the Property $(A)$. Also let $0<\lambda_{h}(f) \leq \rho_{h}(f)<\infty$. Then

$$
\lim _{r \rightarrow \infty} \frac{\log T_{h}^{-1} T_{f \circ g}(r)}{\log T_{M(h)}^{-1} T_{M(f)}\left(\exp r^{\mu}\right)}=\infty,
$$

where $\rho_{g}<\mu<\infty$.

Theorem 28. Let $f$ be a meromorphic function, $g$ be a transcendental entire function either of finite order or of non-zero lower order with $\sum_{a \in \mathbb{C} \cup\{\infty\}} \delta_{1}(a ; g)=4$ and $h$ be a transcendental entire function of regular growth having non zero finite order with $\sum_{a \in \mathbb{C} \cup\{\infty\}} \delta_{1}(a ; h)=4$ and satisfy the Property $(A)$. Also let $\lambda_{h}(g)>0$ and $\rho_{h}(f)<\infty$. Then for every $\mu$ with $\rho_{g}<\mu<\infty$

$$
\lim _{r \rightarrow \infty} \frac{\log T_{h}^{-1} T_{f \circ g}(r)}{\log T_{M(h)}^{-1} T_{M(g)}\left(\exp r^{\mu}\right)}=0 .
$$

\section{Competing interests}

The authors declare that they have no competing interests.

\section{Authors' contributions}

All authors have contributed to all parts of the article. All authors read and approved the final manuscript. 


\section{References}

[1] L. Bernal : Crecimiento relativo de funciones enteras. Contribución al estudio de lasfunciones enteras coníndice exponencial finito, Doctoral Dissertation, University of Seville, Spain, 1984.

[2] L. Bernal : Orden relative de crecimiento de funciones enteras, Collect. Math., Vol. 39 (1988), pp.209-229.

[3] N. Bhattacharjee and I. Lahiri : Growth and value distribution of differential polynomials, Bull. Math. Soc. Sc. Math. Roumanie Tome, Vol. 39(87), No.1-4(1996), pp.85-104.

[4] W. Bergweiler : On the Nevanlinna characteristic of a composite function, Complex Variables, Vol. 10 (1988), pp. $225-236$.

[5] W. Doeringer : Exceptional values of differential polynomials, Pacific J. Math., Vol 98, No.1(1982), pp.55-62.

[6] S. K. Datta : On the order and type of differential monomials, Journal of Pure Mathematics, C.U., Vol.19 (2002), pp.13-17.

[7] S. K. Datta : On the generalized order and generalized type of differential monomials and differential polynomials, Journal of Mechanics of Continua and Mathematical Sciences, Vol. 2, No. 1 (2007), pp.71-79,

[8] S. K. Datta and S. Kar : On the growth of differential monomials, International Journal of Pure and Applied Mathematics (IJPAM), Vol. 45, No. 4 (2008), pp.573-585.

[9] S. K. Datta and S. Kar : Some comparative growth properties of differential polynomials, International Journal of Mathematical Sciences and Engineering Applications (IJMSEA), Vol. 3, No. 1 (2009), pp.113-128.

[10] S. K. Datta and T. Biswas : On the growth of differential polynomials, Wesleyan Journal of Research, Vol. 2, No. 2 (2009), pp.27-37.

[11] S. K. Datta and A. R. Das : Some results on the growth properties of differential monomials, International Mathematical Forum, Vol. 4, No. 38 (2009), pp.1863-1876.

[12] S. K. Datta and E. Jerin : On the growth estimates of differential monomials and differential polynomials generated by meromorphic functions, Journal of Mathematics, N.B.U., Vol. 3 No. 1 (2010), pp.33-52.

[13] S. K. Datta and A. R. Das : Derivation of some results of maximum terms of differential monomials generated by entire functions, Wesleyan Journal of Research, Vol. 4, No. 1 (2011 ) pp. 15-21.

[14] S. K. Datta, A. R. Das and A. R. Maji : Maximum terms of differential monomials generated by entire functions, International Journal of Mathematical Sciences and Engineering Applications (IJMSEA), Vol. 5, No. IV (July, 2011), pp.353-359.

[15] S. K. Datta and A. R. Maji : On different kinds of orders and types of differential monomials generated by transcendental entire functions using their minimum modulus, International Journal of Contemporary Mathematical Sciences, Vol. 6 , No. 42 (2011), pp. 2057 - 2074.

[16] S. K. Datta and A. R. Maji : On different kinds of orders and types of differential polynomials generated by entire functions using their minimum modulus, International Journal of Mathematical Analysis, Vol. 5, No. 43 (2011), pp. 2127 - 2140.

[17] S. K. Datta and A. R. Das : Estimation of growth properties of differential polynomials generated by entire functions on the basis of their maximum terms, Review Bulletin of the Calcutta Mathematical Society, Vol. 19, No. 2 (2011), pp. 193-200.

[18] S. K. Datta and T. Biswas : On a result of Bergweiler, International Journal of Pure and Applied Mathematics (IJPAM), Vol. 51, No. 1 (2009), pp.33-37.

[19] S. K. Datta, T. Biswas and MD. A. Hoque.: On some growth properties of differential polynomials in the light of relative order, Italian Journal of Pure and Applied Mathematics, Vol. N 32 (2014), pp. 235-246..

[20] S. K. Datta, T. Biswas and S. Bhattacharyya : On relative order and relative type based growth properties of differential monomials, Journal of the Indian Math. Soc., Vol. 82, Nos. (3 - 4), (2015), pp. 39-52.

[21] S. K. Datta, T. Biswas and C. Biswas: Measure of growth ratios of composite entire and meromorphic functions with a focus on relative order, International J. of Math. Sci. \& Engg. Appls. (IJMSEA), Vol. 8 No. IV (July, 2014), pp. 207-218.

[22] W. K. Hayman : Meromorphic Functions, The Clarendon Press, Oxford (1964).

[23] B. K. Lahiri and D. Banerjee : Relative order of entire and meromorphic functions, Proc. Nat. Acad. Sci. India Ser. A., Vol. 69(A), No. 3, (1999), pp.339-354.

[24] I. Lahiri : Deficiencies of differential polynomials, Indian J. Pure Appl. Math.,Vol.30, No.5(1999), pp.435-447.

[25] I. Lahiri and S. K. Datta : Growth and value distribution of differential monomials, Indian Journal of Pure and Applied Mathematics, Vol.32, No.12 ( December, 2001), pp.1831-1841.

[26] L.R. Sons : Deficiencies of monomials, Math.Z, Vol.111(1969), pp.53-68.

[27] E. C. Titchmarsh : The theory of functions, 2nd edition, Oxford University Press, Oxford, 1939. 
[28] L. Yang : Value distribution theory and new research on it, Science Press, Beijing (1982).

[29] H. X. Yi : On a result of Singh, Bull. Austral. Math. Soc., Vol. 41(1990), pp.417-420.

[30] G. Valiron : Lectures on the general theory of integral functions, Chelsea Publishing Company, 1949. 